\title{
Paramyxovirus Outbreak in a Long-Term Care Facility: The Challenges of Implementing Infection Control Practices in a Congregate Setting
}

\author{
Steven Schaeffer Spires, MD $;{ }^{1}$ H. Keipp Talbot, MD, MPH; ${ }^{1}$ Carolyn A. Pope, RN, CIC; ${ }^{3}$ Thomas R. Talbot, MD, MPH ${ }^{1,2}$
}

ов јестіVE. We report an outbreak of respiratory syncytial virus (RSV) and human metapneumovirus (HMPV) infections in a dementia care ward containing 2 separately locked units (A and B) to heighten awareness of these pathogens in the older adult population and highlight some of the infection prevention challenges faced during a noninfluenza respiratory viral outbreak in a congregate setting.

Methods. Cases were defined by the presence of new signs or symptoms that included (1) a single oral temperature $\geq 37.8^{\circ} \mathrm{C}\left(100.0^{\circ} \mathrm{F}\right)$ and (2) the presence of at least 2 of the following symptoms: cough, dyspnea, rhinorrhea, hoarseness, congestion, fatigue, and malaise. Attempted infection-control measures included cohorting patients and staff, empiric isolation precautions, and cessation of group activities. Available nasopharyngeal swab specimens were sent to the Tennessee Department of Health for identification by rT-PCR testing.

RESUlts. We identified 30 of the $41(73 \%)$ residents as cases over this 16-day outbreak. Due to high numbers of sick personnel, we were unable to cohort staff to 1 unit. Unit B developed its first case 8 days after infection control measures were implemented. Of the 14 cases with available specimens, 6 patients tested positive for RSV-B, 7 for HMPV and 1 patient test positive for influenza A. Overall, 15 cases (50\%) required transfer to acute care facilities; 10 of these patients (34\%) had chest X-ray confirmed pulmonary infiltrates; and 5 residents (17\%) died.

CONCLUSIONS. This case report highlights the importance of RSV and HMPV in causing substantial disease in the older adult population and highlights the challenges in preventing transmission of these viruses.

Infect Control Hosp Epidemiol 2017;38:399-404

Respiratory syncytial virus (RSV) and human metapneumovirus (HMPV) are 2 closely related enveloped RNA viruses in the Paramyxoviridae family that cause a wide spectrum of disease in older adults ranging from mild upper respiratory tract infections to severe respiratory distress. ${ }^{1-3}$ RSV and HMPV circulate like seasonal influenza viruses and can cause comparable hospitalization rates. ${ }^{4-6}$ Despite the substantial disease morbidity among the older adult population, these infections are still an underrecognized problem likely due to insensitive methods of detection prior to the availability of sensitive molecular testing. ${ }^{7}$

These infections also impact older adults living in long-term care or congregate settings. The first report of nosocomial transmission of RSV was in 1971 on a pediatric ward with subsequent outbreaks an acute and long-term care facilities (LTCF). ${ }^{8-13}$ HMPV has also been associated with several outbreaks in congregate settings since its discovery in 2001.,14-18 Treatment options and vaccines for prevention of RSV and HMPV infections are lacking. Therefore, effective infection prevention measures are necessary to prevent outbreaks of these infections in congregate settings.

We describe an outbreak of respiratory viral illnesses caused by RSV and HMPV in an LTCF among residents with a high rate of influenza vaccination to heighten awareness of these pathogens in the older adult population and highlight some of the infection prevention challenges in an LTCF.

\section{THE OUTBREAK}

The outbreak occurred in 2 locked units (units A and B) that house residents with dementia. The units are separated by a locked door but share clinical staff. Patients cannot move between units without being escorted by healthcare personnel (HCP). Unit A contains 21 double-occupancy rooms and houses residents who need more assistance with activities of daily living (ADL). There is a large resident dining and multipurpose room where most residents commune for meals and other activities throughout the day. Unit B contains 6 double-occupancy rooms,

Affiliations: 1. Department of Medicine, Vanderbilt University School of Medicine, Nashville, Tennessee; 2. Department of Health Policy, Vanderbilt University School of Medicine, Nashville, Tennessee; 3. Division of Nursing, Tennessee Valley Healthcare Administration, Veterans Affairs, Nashville, Tennessee.

Received August 25, 2016; accepted December 1, 2016; electronically published January 9, 2017

(c) 2017 by The Society for Healthcare Epidemiology of America. All rights reserved. 0899-823X/2017/3804-0003. DOI: 10.1017/ice.2016.316 
and the residents are more independent with ADLs but may have a history of aggressive agitation. The rooms on this unit surround a large common area used for dining and regular community activities. The units are located together in a separate wing of a larger facility containing more than 200 long-term care beds. In January 2015, a resident from unit A was transferred to the emergency department (ED) because of a new cough, vomiting, and generalized malaise. His chest radiograph showed an infiltrate, and he was febrile $\left(37.8^{\circ} \mathrm{C}\right.$ or $\left.100.0^{\circ} \mathrm{F}\right)$. On days 2 and 3, 9 more residents from unit A were transferred to the ED or were evaluated by the attending physician with new symptoms of fever, cough, and nasal congestion and/or rhinorrhea. Nasopharyngeal specimens from these patients were negative for influenza by rapid antigen testing. The infectious diseases consultant notified the infection control department (IC) the afternoon of day 3 due to the concern for a respiratory viral outbreak at the facility. After discussion with pertinent leaders, the long-term care unit's influenza outbreak plan, adapted from the CDC's Interim Guidance for Influenza Outbreak Management in Long-Term Care Facilities, was activated. ${ }^{19}$ Prophylactic oseltamivir was prescribed that evening to all nonsymptomatic residents on units $\mathrm{A}$ and $\mathrm{B}$, except for 1 resident who had a history of oseltamivir allergy.

On the morning of day 4, the IC team assisted with an investigation of the outbreak of respiratory illnesses. The pertinent facets of this investigation were as follows.

\section{Case Identification and Surveillance}

Additional information was obtained from medical record review and staff interviews. See Table 1 for signs and symptoms used for case definition. This definition was adopted from the 2008 Infectious Diseases Society of America guideline for evaluating fever and infection in older adults and the consensus guidelines from the Society for Healthcare Epidemiology of America's Long-Term Care Workgroup. ${ }^{20,21}$ The definition for influenza-like illness was modified so staff nurses could elicit the symptoms from patients who were unable to answer questions regarding recent clinical history. We validated our case definition with the first 10 cases that were discovered prior to our active surveillance. The unit charge nurses and midlevel provider

TABLE 1. Case Definition for Active Screening of Residents, Performed Every 12 Hours

\begin{tabular}{l} 
Symptoms \\
- Fever $\geq 37.8^{\circ} \mathrm{C}\left(100.0^{\circ} \mathrm{F}\right)$ \\
- Cough \\
- Ryspnea \\
- Hoarseness \\
- Congestion \\
- Matigue \\
\hline
\end{tabular}

${ }^{\mathrm{a}} \mathrm{A}$ probable case was considered fever plus 2 or more symptoms as listed. assessed all patients for new signs or symptoms at least twice daily throughout the outbreak.

Because this facility did not have on-site polymerase chain reaction (PCR) testing, nasopharyngeal swab specimens from identified cases were sent to the Tennessee Department of Health (TDOH) for real-time reverse-transcription PCR (RT-PCR) testing using a multiplex panel that identified respiratory viruses including influenza A and B, RSV A and B, HMPV, parainfluenza viruses, adenoviruses, and coronaviruses. The results of these tests became available almost 2 weeks after specimen collection.

\section{Infection Control Interventions}

The outbreak infection control plan included the following steps to mitigate respiratory pathogen transmission: (1) cohorting of identified case residents to either private rooms or shared rooms with another case, (2) placement of case residents into droplet and contact precautions (including limitations on travel outside of the patient room and use of personal protective equipment, ie, gowns, gloves, and masks), (3) messaging to staff to avoid working when ill (ie, "presenteeism") and, (4) emphasis on hand hygiene and respiratory etiquette practices, (5) daily leadership huddles including the attending physician, mid-level provider, medical director, and representatives from IC, social work, pharmacy, laboratory, dietary services, environmental services, and nursing, (6) cessation of unit-based group activities, including community meals, (7) visitor restriction, and (8) closure of the unit to new admissions.

\section{RES ULT S}

The epidemiologic investigation found that 30 of the 41 residents were identified with RSV and/or HMPV over this 16-day outbreak. The first case on unit B occurred on day 12 of the outbreak. The overall attack rates were 23 of 31 residents (74\%) on unit A and 7 of 10 residents (70\%) on unit B (Figure 1). Laboratory testing was performed for 14 of the cases: 6 of these residents tested positive for RSV-B, 7 for HMPV and 1 resident tested positive for influenza A. A single resident tested positive for both RSV and HMPV, and only 1 viral panel from the TDOH was negative for all pathogens tested. All patients had been diagnosed with dementia of differing etiologies and severities. They all needed some level of assistance for their ADLs, and none of the patients actively complained to staff of symptoms, likely delaying the recognition of illness. The initial cases were identified because they needed acute medical attention, and later cases were discovered by the formal active surveillance. There were no formal protocols for screening visitors for illnesses prior to this outbreak.

Of the 30 total cases, 15 residents required transfer to acutecare facilities, 10 had chest radiograph-confirmed pulmonary infiltrates, and 5 ( $17 \%$ of the cases, $15 \%$ of all residents on unit A) died in association with this outbreak (Table 2). All 5 deaths were from unit $\mathrm{A}$. Of these deaths, 1 patient was the single 


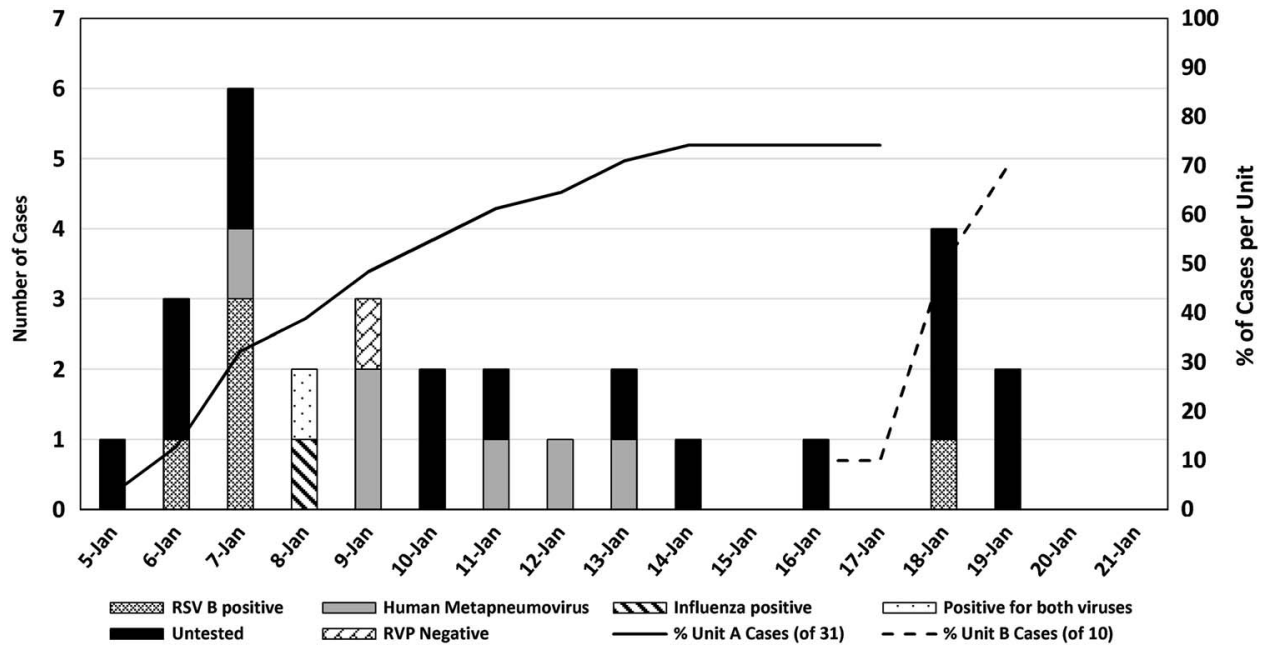

FIGURE 1. Number of cases and positive tests on both units A and B by day. ${ }^{\star}$ RSV, respiratory syncytial virus; RVP, rT-PCR-based respiratory viral panel multiplex.

тавLE 2. Number of Cases per Unit and Their Outcomes

\begin{tabular}{lcccc}
\hline & No. & \% of Cases & Unit A & Unit B \\
\hline Cases, no. (\% of total) & $30(73 \%)$ & $\ldots$ & $23(74 \%)$ & $7(70 \%)$ \\
Transfers to acute-care facilities & 15 & 52 & 11 & 4 \\
Pneumonia, CXR confirmed & 10 & 34 & 7 & 3 \\
Deaths & 5 & 17 & 5 & 0 \\
\hline
\end{tabular}

NOTE. CXR, chest radiograph.

тавце 3. Characteristics of Residents Who Died in Association With This Outbreak

\begin{tabular}{|c|c|c|c|c|c|}
\hline Age, $y$ & $\begin{array}{l}\text { Duration of Symptoms } \\
\text { Before Death, d }\end{array}$ & $\begin{array}{l}\text { Tmax, } \\
{ }^{\circ} \mathrm{C}\left({ }^{\circ} \mathrm{F}\right)\end{array}$ & $\begin{array}{l}\text { Acute Signs and } \\
\text { Symptoms }\end{array}$ & Underlying Chronic Medical Conditions & RVP Results \\
\hline 88 & 5 & $37.77(100.0)$ & $\begin{array}{l}\text { Cough, malaise, } \\
\text { LLL pneumonia }\end{array}$ & Alzheimer's disease, GERD, neurogenic bladder & $\mathrm{N} / \mathrm{T}$ \\
\hline 89 & 3 & $37.88(100.2)$ & $\begin{array}{l}\text { Cough, decreased } \\
\text { appetite }\end{array}$ & $\begin{array}{l}\text { Endstage dementia NOS, prostate cancer, chronic } \\
\text { atrial fibrillation, CKD }\end{array}$ & Influenza A \\
\hline 82 & 14 & $37.88(100.2)$ & Cough, malaise & Dementia NOS, CAD, COPD, chronic dry eyes & HMPV \\
\hline 86 & 14 & $37.94(100.3)$ & $\begin{array}{l}\text { Cough, decreased } \\
\text { appetite }\end{array}$ & $\begin{array}{l}\text { Alzheimer's disease, mood disorder NOS, GERD, } \\
\text { dysphagia }\end{array}$ & $\mathrm{N} / \mathrm{T}$ \\
\hline
\end{tabular}

NOTE. Tmax, maximum recorded oral temperature during the acute illness; LLL, left lower lobe; GERD, gastroesophageal reflux disease; NOS, not otherwise specified; $\mathrm{CKD}$, chronic kidney disease; CAD, coronary artery disease; COPD, chronic obstructive pulmonary disease; DMII, type II diabetes mellitus; N/T, not tested.

influenza case, 1 patient was confirmed positive for HMPV, and 2 patients were already under hospice care for end-stage dementia (Table 3). The mean and median ages of all cases were 81.3 and 84 years, respectively. All of the residents on units A and B had been living at the facility for $>2$ weeks prior to the onset of symptoms.

Even though the unit based midlevel provider and the clinical nurse leader developed an effective system of identifying cases, problems arose in establishing cohorts of these cases and with the attendance of the unit staff. To create cohorts of the affected residents, whenever possible, we moved each symptomatic patient to a private room or to a room housing another case. However, new cases developed quickly, and almost all the residents already had roommates prior to the outbreak, leaving only a few singly occupied rooms available. When a new case developed, that patient was placed 
with another resident with a known case in an initial attempt to isolate cases in a cohort. Occasionally, the bed recently voided would then be filled with another asymptomatic resident due to the lack of extra rooms. The method of bed tracking was paper based, and some patients were transferred to different rooms more than once per day, which made it challenging to track patient movement with the current system. No residents were transferred from unit A to unit B within the 2 weeks prior to or during the outbreak.

Messaging to HCP to avoid working when ill was attempted; however, during the first week of the outbreak, one-third of the nursing pool either called in sick or were sent home due to symptoms consistent with a probable case. The extensive reductions in the work force during this respiratory viral season made effective cohort isolation of HCP logistically impossible, and the 2 units ultimately had to share HCP. Many HCP continued to work while ill and wore surgical masks throughout the outbreak. Due to policies attempting to protect HCP privacy, IC was prohibited from actively screening HCP.

While all cases were placed on contact and droplet precautions, this intervention also proved challenging. Due to dementia, many patients did not adhere to the recommended restrictions. PPE availability became an issue during the second week of the outbreak when the number of rooms or areas that needed an isolation cart exceeded the number of available carts. During a short period at the end of the first week, the facility's supply of isolation gowns and masks was exhausted; however, this shortage was remedied quickly by a rush order from a local supplier.

Prior to the outbreak, alcohol-based hand rub (ABHR) containers could not be placed at the convenient locations because of prior ingestion of the ABHR by a resident. Each room did have accessible sinks, and after the outbreak was recognized, the staff were given personal bottles of ABHR to carry on their uniforms.

\section{I S C US S I O N}

Management of this outbreak was very challenging, with logistic issues notably related to the congregate setting, residents with dementia, and significant staff reductions during this respiratory viral season.

Considering this experience, we identified 3 principle methods necessary to prevent future outbreaks of a respiratory virus within our LTCF. These included (1) effective surveillance for cases among residents and HCP during the respiratory viral season and an efficient method for identifying potential contagious pathogens rapidly, (2) effective and real-time, data-driven methods for isolating patients and staff in cohort groups in resource-limited settings, and (3) strict adherence to hand hygiene and use of appropriate isolation precautions.

Early detection of a contagious pathogen and identification of infected patients is important when trying to prevent an outbreak. Most LTCF residents are not formally examined by a medical provider daily, and they may not be able to provide an adequate history of new symptoms due to underlying comorbid illness such as dementia. To address this challenge, a daily active screening process now occurs for the residents and visitors during the respiratory viral season. Notably, our outbreak case definition required fever, albeit lowered to $37.8^{\circ} \mathrm{C}\left(100.0^{\circ} \mathrm{F}\right)$, largely because the known cases had evidence of elevated temperature and we sought to identify an influenza-like illness. However, while trying to maintain some specificity, we may have missed residents with upper respiratory illness who did not mount a robust enough febrile response for us to measure. Thus, during active surveillance for a possible respiratory viral infection, we recommend simply allowing it to be one of several possible signs rather than a required symptom. ${ }^{20}$ Determining the etiologic agent of an outbreak will help guide the methods of prevention, including the use of chemoprophylaxis if available. Respiratory viral testing results are now available for this facility within 24-48 hours through a collaboration with a private laboratory at a local hospital to perform rT-PCR testing using a multiplex panel.

Once cases are identified, effective cohort isolation of residents and staff is also paramount to halting transmission. ${ }^{22-25}$ Many residents in LTCFs share rooms and HCP staffing can be limited; thus, when establishing cohorts during an outbreak of a respiratory viral pathogen, we suggest treating the room with a case plus exposed roommate as a single unit in terms of isolation. This approach prevents switching of roommates and allows for cohort isolation to a particular wing or hall within the affected unit, which also achieves more effective cohort isolation of staff. Notably, we did not use this method of cohort isolation and cannot speak to its efficacy. In addition, if a patient is moved from their shared room to a private bed, then caution must be taken to prevent filling the newly vacated bed with a separate asymptomatic, unexposed resident. Such replacement can theoretically help propagate the transmission of the virus.

In LTCFs, the transmission of pathogens can often be epidemiologically linked to HCP who unwittingly serve as vectors carrying pathogens from one patient to another. ${ }^{9,10,25-27}$ Many HCP may not develop the same clinical manifestations from a particular infection (eg, may be asymptomatic), but they can effectively transmit the infection while working. ${ }^{28}$ As a result, we recommend that HCP remain either with the isolated cohort or the uninfected group throughout the entire outbreak.

Because different viruses have been shown to have varying methods of transmission, empiric contact and droplet precautions are recommended for residents in this congregate setting with a febrile respiratory illness until an etiology is determined. Reports from acute-care facilities indicate that when hand hygiene and isolation precautions are effectively adopted, the transmission of selected respiratory viruses between HCP and patients is limited. ${ }^{25,29}$ Any resident who is intolerant or nonadherent to the recommended isolation precautions should wear a surgical mask when leaving the 
room, should adhere to appropriate respiratory etiquette, and should perform hand hygiene procedures. We emphasize that staff monitor residents' compliance to these precautions and that nonadherence should be addressed immediately with infection control representatives, the hospital epidemiologist, and the attending provider.

As mentioned earlier, the ABHR containers had been removed from the convenient locations due to a previous resident who had a history of alcoholism and had ingested the ethanol-containing antiseptic. This is a known concern that has been documented in the literature. ${ }^{30,31}$ However, this scenario occurred several years ago, and this resident has since left. Since the outbreak, this particular wing of the facility has been renovated and refurbished with hand rub dispensers at every doorway entrance. Staff also monitor for oral ingestion by the residents.

In conclusion, this outbreak was a sentinel event for our institution that houses more than 200 long-term care beds. Identification of infected residents and HCP as well as implementation of essential infection prevention strategies were challenges that are likely not unique to our specific LTCF. Since this outbreak, several additions and changes to the facility's infection prevention procedures that are aimed to prevent spread of all respiratory pathogens in a congregate setting instead of just focused on influenza have been implemented. These improvements have also been accompanied by intensive efforts aimed at improving the overall culture of safety.

\section{ACKNOWLEDGMENTS}

The authors thank Dr William Schaffner for his thoughtful review of this manuscript.

Financial support: No financial support was provided relevant to this article. Potential conflicts of interest: H.K.T. reports having been a consultant for VaxInnate, Novartis, and Gilead and having received grant support from CDC, NIH, MedImmune, and Sanofi.

Address correspondence to Steven Schaeffer Spires, MD, Assistant Professor, Division of Infectious Diseases, Vanderbilt University School of Medicine, A2200 MCN, $116121^{\text {st }}$ Ave South, Nashville, TN 37232-2605 (steven.s.spires@vanderbilt.edu).

\section{REFERENCES}

1. Hall CB, Simoes EA, Anderson LJ. Clinical and epidemiologic features of respiratory syncytial virus. Curr Top Microbiol Immunol 2013;372:39-57.

2. Mathur U, Bentley DW, Hall CB. Concurrent respiratory syncytial virus and influenza $A$ infections in the institutionalized elderly and chronically ill. Ann Intern Med 1980;93:49-52.

3. Liao RS, Appelgate DM, Pelz RK. An outbreak of severe respiratory tract infection due to human metapneumovirus in a long-term care facility for the elderly in Oregon. J Clin Virol 2012;53:171-173.

4. Widmer K, Zhu Y, Williams JV, Griffin MR, Edwards KM, Talbot HK. Rates of hospitalizations for respiratory syncytial virus, human metapneumovirus, and influenza virus in older adults. J Infect Dis 2012;206:56-62.
5. Widmer K, Griffin MR, Zhu Y, Williams JV, Talbot HK. Respiratory syncytial virus- and human metapneumovirus-associated emergency department and hospital burden in adults. Influenza Other Respir Viruses 2014;8:347-352.

6. Ellis SE, Coffey CS, Mitchel EF Jr, Dittus RS, Griffin MR. Influenza- and respiratory syncytial virus-associated morbidity and mortality in the nursing home population. J Am Geriatr Soc 2003;51:761-767.

7. Branche AR, Falsey AR. Respiratory syncytial virus infection in older adults: an under-recognized problem. Drugs Aging 2015; 32:261-269.

8. Ditchburn RK, McQuillin J, Gardner PS, Court SD. Respiratory syncytial virus in hospital cross-infection. Br Med J 1971;3: 671-673.

9. Hall CB, Douglas RG Jr, Geiman JM, Messner MK. Nosocomial respiratory syncytial virus infections. N Engl J Med 1975;293: 1343-1346.

10. Hall CB, Douglas RG Jr, Geiman JM. Possible transmission by fomites of respiratory syncytial virus. J Infect Dis 1980;141: 98-102.

11. Hall CB. Respiratory syncytial virus: its transmission in the hospital environment. Yale J Biol Med 1982;55:219-223.

12. Caram LB, Chen J, Taggart EW, et al. Respiratory syncytial virus outbreak in a long-term care facility detected using reverse transcriptase polymerase chain reaction: an argument for real-time detection methods. J Am Geriatr Soc 2009;57: 482-485.

13. Sorvillo FJ, Huie SF, Strassburg MA, Butsumyo A, Shandera WX, Fannin SL. An outbreak of respiratory syncytial virus pneumonia in a nursing home for the elderly. J Infect 1984;9:252-256.

14. Honda H, Iwahashi J, Kashiwagi T, et al. Outbreak of human metapneumovirus infection in elderly inpatients in Japan. J Am Geriatr Soc 2006;54:177-180.

15. Neu N, Plaskett T, Hutcheon G, Murray M, Southwick KL, Saiman L. Epidemiology of human metapneumovirus in a pediatric long-term care facility. Infect Control Hosp Epidemiol 2012;33:545-550.

16. McCracken JP, Arvelo W, Ortiz J, et al. Comparative epidemiology of human metapneumovirus- and respiratory syncytial virusassociated hospitalizations in Guatemala. Influenza Other Respir Viruses 2014;8:414-421.

17. Boivin G, De Serres G, Hamelin ME, et al. An outbreak of severe respiratory tract infection due to human metapneumovirus in a long-term care facility. Clin Infect Dis 2007;44:1152-1158.

18. Louie JK, Schnurr DP, Pan CY, et al. A summer outbreak of human metapneumovirus infection in a long-term-care facility. J Infect Dis 2007;196:705-708.

19. Interim Guidance for influenza outbreak management in long term care facilities. Centers for Disease Control and Prevention website. http://www.cdc.gov/flu/professionals/infectioncontrol/ ltc-facility-guidance.htm. Published 2016. Accessed December 5, 2016.

20. Stone ND, Ashraf MS, Calder J, et al. Surveillance definitions of infections in long-term care facilities: revisiting the McGeer criteria. Infect Control Hosp Epidemiol 2012;33:965-977.

21. High KP, Bradley SF, Gravenstein S, et al. Clinical practice guideline for the evaluation of fever and infection in older adult residents of long-term care facilities: 2008 update by the Infectious Diseases Society of America. J Am Geriatr Soc 2009; 57:375-394. 
22. Gala CL, Hall CB, Schnabel KC, et al. The use of eye-nose goggles to control nosocomial respiratory syncytial virus infection. JAMA 1986;256:2706-2708.

23. Gaspard P, Mosnier A, Cohen JM, et al. [Clusters of respiratory tract infections and alert strategy in nursing homes]. Med Mal Infect 2011;41:253-261.

24. Liu JW, Lu SN, Chen SS, et al. Epidemiologic study and containment of a nosocomial outbreak of severe acute respiratory syndrome in a medical center in Kaohsiung, Taiwan. Infect Control Hosp Epidemiol 2006;27:466-472.

25. Madge P, Paton JY, McColl JH, Mackie PL. Prospective controlled study of four infection-control procedures to prevent nosocomial infection with respiratory syncytial virus. Lancet 1992;340:1079-1083.

26. Kassis C, Champlin RE, Hachem RY, et al. Detection and control of a nosocomial respiratory syncytial virus outbreak in a stem cell transplantation unit: the role of palivizumab. Biol Blood Marrow Transplant 2010;16:1265-1271.

27. Rainwater-Lovett K, Chun K, Lessler J. Influenza outbreak control practices and the effectiveness of interventions in long-term care facilities: a systematic review. Influenza Other Respir Viruses 2014;8:74-82.

28. Bradley SF. Prevention of influenza in long-term-care facilities. Long-Term-Care Committee of the Society for Healthcare Epidemiology of America. Infect Control Hosp Epidemiol 1999;20:629-637.

29. Stupica D, Lusa L, Petrovec M, et al. Respiratory viruses in patients and employees in an intensive care unit. Infection 2012;40:381-388.

30. Roberts HS, Self RJ, Coxon M. An unusual complication of hand hygiene. Anaesthesia 2005;60:100-101.

31. Meyer P, Baudel JL, Maury E, Offenstadt G. A surprising side effect of hand antisepsis. Intensive Care Med 2005;31:1600. 